\title{
On Solutions to Euler-Lagrange Equations Governing Isotropic, Homogeneous, Naturally Curved Kirchhoff's Elastic Rods
}

\author{
Li-Tien Cheng • Kai Hu
}

Received: 30 July 2008 / Published online: 12 May 2010

(C) The Author(s) 2010. This article is published with open access at Springerlink.com

\begin{abstract}
Motivated by the elastic rod model for DNA with intrinsic curvature, we study the solution space of the Euler-Lagrange equations governing isotropic, homogeneous, naturally curved Kirchhoff's elastic thin rods. Our studies show that for each given total energy and twisting density, there are at most three solutions, aside from the case where the twisting density is some particular constant. We also propose in this paper a reasonable condition under which an improvement on the number of the solutions may be possible. Finally, numerical calculations are presented to support our conclusions.
\end{abstract}

Keywords Naturally curved rods · Total energy $\cdot$ Twisting density $\cdot$ Elastic rod model for DNA · DNA with intrinsic curvature

Mathematics Subject Classification (2000) 74B05 · 74B10 - 74G35 · 74K10 • 92C40 • 92E10

\section{Introduction}

Kirchhoff's theory of isotropic, homogeneous, elastic thin rods has been employed to study the configurations of DNA, which play an important role in genome organization, replication, transcription, and recombination (there is a detailed description of how to treat DNA as an elastic rod in [34]). In the beginning, owing to Watson-Crick model [33], these rods were assumed to be naturally straight; see, for example, [3-7, 11-14, 16, 19, 26, 27, 29, 32] and the references therein. Subsequently, DNAs with intrinsic curvature were substantially documented: either occurring naturally $[22,38]$ or synthesized artificially [31]. Therefore,

\section{L.-T. Cheng}

Department of Mathematics, University of California, San Diego, La Jolla, CA 92093-0112, USA

e-mail: lcheng@math.ucsd.edu

K. Hu (凶)

Department of Applied Mathematics, National Dong Hwa University, Hualien, Taiwan 97401,

Republic of China

e-mail: khu@mail.ndhu.edu.tw 
elastic rods modelling such DNAs do not have a straight undeformed state any longer; see, for example, [10, 21, 23-25, 28, 30, 34-37] and the references therein. In this paper, we focus on these naturally curved elastic rods. As a matter of fact, the axis of their undeformed state is restricted to a circular arc (including, a circle) or a circular helix so that KirchhoffClebsch conservation law of total energy [20] is sustained.

In order to obtain detailed information on DNA tertiary structures, one needs to solve Euler-Lagrange equations that govern elastic rods (the equations are derived from the elastic energy functional and appropriate conditions idealizing practical situations). This was successful in the case of naturally straight rods: the Euler-Lagrange equations have closed-form solutions, owing to the existence of two first integrals; see, (10) and (11) in [18]. Unfortunately, solving the Euler-Lagrange equations for naturally curved equilibrium rods is more difficult.

The procedure for solving the Euler-Lagrange equations for naturally straight equilibrium rods is to first get constant twisting density, and next to use the values of the aforementioned first integrals to solve for the axis curvature of the rods [18]. Therefore, we may conclude that there are infinitely many solutions with the same total energy and twisting density. We would like to investigate if this conclusion still holds in the case of naturally curved rods. That is, we seek the answer to the following problem:

Multiplicity Problem How many solutions to the Euler-Lagrange equations governing naturally curved rods have the same total energy and twisting density?

Before reporting the result of our investigation, let $\rho, \tilde{\rho}$ and $t_{w}$ denote the bending stiffness, twisting stiffness and the twisting density of a rod, respectively (see [15] for the stiffnesses from the viewpoint of biological physics; both stiffnesses are constant), and let $\tau$ be the axis geometric torsion of the undeformed state of the rod.

Theorem 1 Suppose $t_{w}$ is constant. If $(2 \rho-\tilde{\rho}) t_{w}+\tilde{\rho} \tau \neq 0$, then there are at most three solutions with the same total energy and twisting density. However, if $(2 \rho-\tilde{\rho}) t_{w}+\tilde{\rho} \tau=0$, then there are infinitely many solutions.

Theorem 2 There are at most three solutions with the same total energy and twisting density, provided the latter is nonconstant.

Our result suggests that the elasticity of naturally straight rods is very unique: as the axis curvature of the undeformed state starts increasing from zero, the number of solutions with the same total energy and twisting density is reduced greatly, and solutions with nonconstant twisting density emerge.

The idea of our proof originates from the meaning of one of the Euler-Lagrange equations: the twisting density $t_{w}$ of each naturally curved equilibrium rod determines one of the bending curvatures $k_{1}$ of the rod. It is thus crucial to count the number of distinct $k_{2}$ 's, the other bending curvature, of equilibrium rods with the same total energy and twisting density. Since no two distinct $k_{2}$ 's can have the same data: $k_{2}\left(s_{0}\right)$ and $\dot{k}_{2}\left(s_{0}\right)$ where $s_{0} \in[0, L]$ and $L$ is the length of the rod axis (see Proposition 2), this is equivalent to counting the number of data at a preferred point chosen according to some particular properties of the twisting density.

One might think that the values of $k_{2}\left(s_{0}\right)$ and $\dot{k}_{2}\left(s_{0}\right)$ may be arbitrary. This, however, is nearly incorrect since the Euler-Lagrange equations induce a dependence of $\dot{k}_{2}\left(s_{0}\right)$ on $k_{2}\left(s_{0}\right)$ (when twisting density is not constant). Perhaps, it is more surprising to acknowledge that 
$k_{2}\left(s_{0}\right)$ itself cannot be arbitrary either because it must be a root of a cubic equation derived from the Euler-Lagrange equations (the cubic equation becomes void for the second case of Theorem 1); see Proposition 3 for details.

This paper is organized as follows. Section 2 reviews Kirchhoff's elasticity theory of thin rods. Section 3 presents a proof of Theorem 1. Theorem 2 is proved in Sect. 4. In Sect. 5, we discuss a possible improvement of Theorem 2 under a special condition that is widely assumed in the elastic rod model for DNA structures. A few numerical calculations supporting the improvement are also demonstrated in the last section.

\section{Preliminaries}

In addition to being cylindrical and slender, the configuration of any elastic rod studied in this paper is assumed to be completely determined by an immersed curve $C$, called the axis, and a unit normal vector field $\boldsymbol{v}$ defined along $C$, called the material direction. We further demand that all the rods are inextensible and unshearable, and that they have the same undeformed state $\mathcal{R}_{u}$.

The meaning of ends is clear for open rods, but ambiguous for closed rods. One way to resolve the ambiguity is to mark a normal cross section such that two sides of the cross section are considered ends of a closed rod. This resolution originates from [2] in which the authors studied terminal twist induced writhe of elastic rings through a cut-rotate-seal procedure performed on a normal cross section of the rings (type I topoisomerases change topology of closed circular DNA by using a mechanism equivalent to this procedure [1]). For any rod, we always assume that the arc length parameter $s$ of the rod axis attains the value $L$ at the point on the axis that belongs to the normal cross section on which the twist exerts.

An elastic rod gives rise to the so-called directors $\mathbf{d}_{1}, \mathbf{d}_{2}$ and $\mathbf{d}_{3}$, where $\mathbf{d}_{1}=\boldsymbol{v}, \mathbf{d}_{3}$ is the unit vector tangent to $C$ and $\mathbf{d}_{2}=\mathbf{d}_{3} \times \mathbf{d}_{1}$. Using rigid body motions of $\mathbb{R}^{3}$, we may always assume that for each rod the axis is at the origin of $\mathbb{R}^{3}$ when $s=0$, and $\mathbf{d}_{1}(0), \mathbf{d}_{2}(0)$ and $\mathbf{d}_{3}(0)$ are respectively the standard basis vectors $\mathbf{i}, \mathbf{j}$ and $\mathbf{k}$ of $\mathbb{R}^{3}$. The infinitesimal changes of the directors along $C$ are encoded in the Darboux vector $\mathbf{u}$ as follows:

$$
\frac{d \mathbf{d}_{i}}{d s}=\mathbf{u} \times \mathbf{d}_{i} \quad \text { for } i=1,2,3 .
$$

Let $\mathbf{u}$ be written as $\mathbf{u}=\sum_{i=1}^{3} u_{i} \mathbf{d}_{i}$, then

$$
u_{1}=-\frac{d \mathbf{d}_{3}}{d s} \cdot \mathbf{d}_{2}, \quad u_{2}=\frac{d \mathbf{d}_{3}}{d s} \cdot \mathbf{d}_{1} \quad \text { and } \quad u_{3}=\frac{d \mathbf{d}_{1}}{d s} \cdot \mathbf{d}_{2}
$$

Here, the dot between $d \mathbf{d}_{i} / d s$ and $\mathbf{d}_{j}$ is the standard inner product of $\mathbb{R}^{3}$. The $u_{i}$ of $\mathcal{R}_{u}$ is in particular denoted by $\sigma_{i}$ for $i=1,2,3$.

The elastic energy functional defined for rods is

$$
\frac{1}{2} \int_{0}^{L} \rho\left(u_{1}-\sigma_{1}\right)^{2}+\rho\left(u_{2}-\sigma_{2}\right)^{2}+\tilde{\rho}\left(u_{3}-\sigma_{3}\right)^{2} d s .
$$

A rod $\mathcal{R}$ is called an elastica if it is an equilibrium of the elastic energy functional among all the rods obtained from applying infinitesimal perturbations to $\mathcal{R}$ that neither move nor 
rotate the ends of the rod. Therefore, the $u_{i}$ 's of $\mathcal{R}$ satisfy the following Euler-Lagrange equations:

$$
\begin{aligned}
\rho \dot{u}_{1}+(\tilde{\rho}-\rho) u_{2} u_{3} & =\boldsymbol{l}_{\mathcal{R}} \cdot \mathbf{d}_{2}+\rho \dot{\sigma}_{1}+\tilde{\rho} u_{2} \sigma_{3}-\rho u_{3} \sigma_{2}, \\
\rho \dot{u}_{2}+(\rho-\tilde{\rho}) u_{1} u_{3} & =-\boldsymbol{l}_{\mathcal{R}} \cdot \mathbf{d}_{1}+\rho \dot{\sigma}_{2}-\tilde{\rho} u_{1} \sigma_{3}+\rho u_{3} \sigma_{1}, \\
\tilde{\rho} \dot{u}_{3} & =\tilde{\rho} \dot{\sigma}_{3}+\rho u_{1} \sigma_{2}-\rho u_{2} \sigma_{1}
\end{aligned}
$$

where $\boldsymbol{l}_{\mathcal{R}}$ is some rod-dependent constant vector.

The preceding equations are derived by means of a rather unusual method involving ideas of Riemannian geometry; see [17] for details. Briefly speaking, the directors of a rod $\mathcal{R}$ give rise to a curve $q$ on $S^{3}$ starting at $(1,0,0,0)$, while comparing with $\mathbf{i}, \mathbf{j}, \mathbf{k}[17$, Sect. II] (or see Appendix), where $S^{3}=\left\{\mathbf{q}=\left(q_{1}, q_{2}, q_{3}, q_{4}\right) \in \mathbb{R}^{4}:\|\mathbf{q}\|=1\right\}$. Through this correspondence, an end-fixing variation of $q$ on $S^{3}$ means an inextensible and unshearable perturbation which neither rotates both ends of $\mathcal{R}$ simultaneously nor moves the end of $\mathcal{R}$ corresponding to $s=0$ [17, Lemma 1]. Therefore, Lagrange multipliers are used to capture the restriction left behind, that is, the end of $\mathcal{R}$ corresponding to $s=L$ does not move (these multipliers are written in vector form $\boldsymbol{l}_{\mathcal{R}}$ ). In the upshot, through a series of calculations employing geometry of some conformal $S^{3}$ induced by the stiffnesses $\rho$ and $\tilde{\rho}$, the equations are obtained [17, Sect. IIIB]. Since the $u_{i}$ 's of $\mathcal{R}$ are the components of the tangent vector of $q$ with respect to some frame naturally chosen on $S^{3}$, (1)-(3) can be considered second-order differential equations of the $q_{i}$ 's (this also can be concluded by observing (18) in Appendix).

The sum of $\left(\rho u_{1}^{2}+\rho u_{2}^{2}+\tilde{\rho} u_{3}^{2}\right) / 2$ and $\boldsymbol{l}_{\mathcal{R}} \cdot \mathbf{d}_{3}$ is not always constant for any elastica, unless all the $\sigma_{i}$ 's are constants [20]. The condition on the $\sigma_{i}$ 's limits the configuration of $\mathcal{R}_{u}$ : the axis $C_{u}$ is a line, a circular arc, or a circular helix, and the material direction $\boldsymbol{v}_{u}$ differs from the principal normal vector field $\mathbf{n}_{u}$ of the axis $C_{u}$ by a constant angle (note, when $C_{u}$ is a line, assumed to be lying on the $z$-axis, we choose $(1,0,0)$ to be the $\mathbf{n}_{u}$ since the latter is not defined in this case).

In order to study elastic rods with a rather general undeformed state, for example, only the axis is restricted to one of the aforementioned curves, let $\phi$ be the angle measured from $\mathbf{n}_{u}$ to $\boldsymbol{v}_{u}$, with respect to the orientation represented by the unit tangent vector field of $C_{u}$, at each normal cross section; we may further assume $\phi(0)=0$ so that $\phi$ can be simply written as $\phi(s)=\int_{0}^{s} \sigma_{3} d s$. For any rod, define

$$
\mathbf{e}_{1}=\mathbf{d}_{1} \cos \phi-\mathbf{d}_{2} \sin \phi, \quad \mathbf{e}_{2}=\mathbf{d}_{1} \sin \phi+\mathbf{d}_{2} \cos \phi \quad \text { and } \quad \mathbf{e}_{3}=\mathbf{d}_{3} .
$$

The bending curvatures $k_{1}, k_{2}$ and the twisting density $t_{w}$ of the rod are defined by

$$
k_{1}=-\frac{d \mathbf{e}_{3}}{d s} \cdot \mathbf{e}_{2}, \quad k_{2}=\frac{d \mathbf{e}_{3}}{d s} \cdot \mathbf{e}_{1} \quad \text { and } \quad t_{w}=\frac{d \mathbf{e}_{1}}{d s} \cdot \mathbf{e}_{2} .
$$

Then,

$$
k_{1}=u_{1} \cos \phi-u_{2} \sin \phi, \quad k_{2}=u_{1} \sin \phi+u_{2} \cos \phi \quad \text { and } \quad t_{w}=u_{3}-\dot{\phi} .
$$

Using (5), the elastic energy functional becomes

$$
\frac{1}{2} \int_{0}^{L} \rho k_{1}^{2}+\rho\left(k_{2}-\kappa\right)^{2}+\tilde{\rho}\left(t_{w}-\tau\right)^{2} d s,
$$


where $\kappa$ and $\tau$ are the curvature and the geometric torsion of $C_{u}$, respectively. Furthermore, using (4), (5) and the fact that $\dot{\phi}=\sigma_{3},(1)-(3)$ can be rewritten as

$$
\begin{aligned}
\rho \dot{k}_{1}+(\tilde{\rho}-\rho) k_{2} t_{w}-\tilde{\rho} \tau k_{2}+\rho \kappa t_{w} & =\boldsymbol{l}_{\mathcal{R}} \cdot \mathbf{e}_{2}, \\
\rho \dot{k}_{2}+(\rho-\tilde{\rho}) k_{1} t_{w}+\tilde{\rho} \tau k_{1} & =-\boldsymbol{l}_{\mathcal{R}} \cdot \mathbf{e}_{1}, \\
\tilde{\rho} \dot{t}_{w} & =\rho \kappa k_{1},
\end{aligned}
$$

and vice versa. This suggests that any elastica is a rod whose bending curvatures and twisting density satisfy (6)-(8).

Because $\mathbf{d}_{1}$ and $\mathbf{d}_{2}$ depend on $q$, the terms $\boldsymbol{l}_{\mathcal{R}} \cdot \mathbf{e}_{1}$ and $\boldsymbol{l}_{\mathcal{R}} \cdot \mathbf{e}_{2}$ are functions of the $q_{i}$ 's. Hence, (6) and (7) are not differential equations of $k_{i}$ 's, and we will not solve them for the $k_{i}$ 's. Instead, for a given elastica $\mathcal{R}$, we write $\boldsymbol{l}_{\mathcal{R}}=-\lambda_{1} \mathbf{e}_{1}+\lambda_{2} \mathbf{e}_{2}+\lambda_{3} \mathbf{e}_{3}$. Since $\boldsymbol{l}_{\mathcal{R}}$ is constant, $\dot{\boldsymbol{l}}_{\mathcal{R}}=\mathbf{0}$ and this gives

$$
\begin{aligned}
\dot{\lambda}_{1}+\lambda_{2} t_{w}-\lambda_{3} k_{2} & =0, \\
-\lambda_{1} t_{w}+\dot{\lambda}_{2}-\lambda_{3} k_{1} & =0, \\
\lambda_{1} k_{2}+\lambda_{2} k_{1}+\dot{\lambda}_{3} & =0 .
\end{aligned}
$$

Using (6) and (7) which respectively express $\lambda_{2}$ and $\lambda_{1}$ in terms of the bending curvatures and twisting density of the elastica $\mathcal{R},(11)$ becomes

$$
\rho k_{1} \dot{k}_{1}+\rho k_{2} \dot{k}_{2}+\rho \kappa k_{1} t_{w}+\dot{\lambda}_{3}=0 .
$$

By virtue of (8), we rediscover the so-called Kirchhoff-Clebsch conservation law [20], stating that the function

$$
\frac{\rho}{2}\left(k_{1}^{2}+k_{2}^{2}\right)+\frac{\tilde{\rho}}{2} t_{w}^{2}+\lambda_{3},
$$

called the total energy of the elastica $\mathcal{R}$, is always constant. We denote this constant by $\epsilon$ from here on.

For the rest of this paper, we study the $k_{i}$ 's of an elastica through (8)-(10) where $\lambda_{1}, \lambda_{2}$ and $\lambda_{3}$ are respectively replaced by

$$
\rho \dot{k}_{2}+(\rho-\tilde{\rho}) k_{1} t_{w}+\tilde{\rho} \tau k_{1}, \quad \rho \dot{k}_{1}+(\tilde{\rho}-\rho) k_{2} t_{w}-\tilde{\rho} \tau k_{2}+\rho \kappa t_{w}
$$

and

$$
\epsilon-\frac{\rho}{2}\left(k_{1}^{2}+k_{2}^{2}\right)-\frac{\tilde{\rho}}{2} t_{w}^{2}
$$

for some constant $\epsilon$. If $\mathbf{x}$ denotes the transpose of the vector-valued function $\left(k_{1}, k_{2}, t_{w}\right.$, $\left.\dot{k}_{1}, \dot{k}_{2}\right)$ defined on $[0, L]$, then (8)-(10) can be rewritten as $\dot{\mathbf{x}}=\mathbf{F}(\mathbf{x})$ where

$$
\mathbf{F}(\mathbf{x})=\left(\begin{array}{c}
\dot{k}_{1} \\
\dot{k}_{2} \\
\frac{\rho \kappa}{\tilde{\rho}} k_{1} \\
\frac{2 \rho-\tilde{\rho}}{\rho} t_{w} \dot{k}_{2}+\frac{\tilde{\rho} \tau}{\rho} \dot{k}_{2}+k_{1}\left(\frac{\epsilon}{\rho}-\frac{1}{2}\left(k_{1}^{2}+k_{2}^{2}\right)+\frac{2 \rho-3 \tilde{\rho}}{2 \rho} t_{w}^{2}\right)+\frac{\kappa(\rho-\tilde{\rho})}{\tilde{\rho}} k_{1} k_{2}+\frac{\tilde{\rho} \tau}{\rho} k_{1} t_{w}-\frac{\rho \kappa^{2}}{\tilde{\rho}} k_{1} \\
\frac{\tilde{\rho}-2 \rho}{\rho} t_{w} \dot{k}_{1}-\frac{\tilde{\rho} \tau}{\rho} \dot{k}_{1}+k_{2}\left(\frac{\epsilon}{\rho}-\frac{1}{2}\left(k_{1}^{2}+k_{2}^{2}\right)+\frac{2 \rho-3 \tilde{\rho}}{2 \rho} t_{w}^{2}\right)+\frac{\kappa(\tilde{\rho}-\rho)}{\tilde{\rho}} k_{1}^{2}+\frac{\tilde{\rho} \tau}{\rho} k_{2} t_{w}-\kappa t_{w}^{2}
\end{array}\right) .
$$


Proposition 1 The bending curvatures and the twisting density of an elastica are real analytic. Therefore, the pre-image of any number under any one of the functions is a finite subset of $[0, L]$ if the function is not constant.

Proof Because $\mathbf{F}$ is a real analytic vector-valued function of $\mathbf{x}$, any solution to $\dot{\mathbf{x}}=\mathbf{F}(\mathbf{x})$ is real analytic [8]. As a result, $k_{1}, k_{2}$ and $t_{w}$ are real analytic. Since the pre-image of any number under a nonconstant real analytic function has no limit point and $[0, L]$ is compact, it is finite.

Because $t_{w}$ determines $k_{1}$ via (8), we sometimes use a triplet $\left(\epsilon, k_{2}, t_{w}\right)$ to denote a solution to (8)-(10) with total energy $\epsilon$.

Proposition 2 Let $\left(\epsilon, k_{2}, t_{w}\right)$ and $\left(\epsilon, \widetilde{k}_{2}, t_{w}\right)$ denote two solutions. If there exists a point $s_{0}$ satisfying $k_{2}\left(s_{0}\right)=\widetilde{k}_{2}\left(s_{0}\right)$ and $\dot{k}_{2}\left(s_{0}\right)=\dot{\widetilde{k}}_{2}\left(s_{0}\right)$, then $k_{2}$ and $\widetilde{k}_{2}$ are identical. Namely, if $u=k_{2}-\widetilde{k}_{2}$, then $u^{2}+(\dot{u})^{2}$ is either zero or positive over $[0, L]$.

Proof We first rewrite (9) as

$$
\begin{aligned}
\ddot{k}_{2}= & \frac{\tilde{\rho}(\tilde{\rho}-2 \rho)}{\rho^{2} \kappa} t_{w} \ddot{t}_{w}+\frac{\tilde{\rho}(\tilde{\rho}-\rho)}{\rho^{2} \kappa}\left(\dot{t}_{w}\right)^{2}-\kappa t_{w}^{2}-\frac{\tilde{\rho}^{2}}{\rho^{2} \kappa} \tau \ddot{t}_{w}+\frac{\tilde{\rho}}{\rho} \tau k_{2} t_{w} \\
& +\frac{1}{\rho} k_{2}\left(\epsilon-\frac{\rho}{2} k_{2}^{2}-\frac{\tilde{\rho}^{2}}{2 \rho \kappa^{2}}\left(\dot{t}_{w}\right)^{2}+\frac{2 \rho-3 \tilde{\rho}}{2} t_{w}^{2}\right) .
\end{aligned}
$$

Then it is easy to see that the higher-order derivatives of $k_{2}$ are determined by its lower-order derivatives (and, of course, also by $\rho, \tilde{\rho}, \kappa, t_{w}$ and $t_{w}$ 's derivatives that we suppress at the moment). So if there are two solutions satisfying the hypotheses, then $k_{2}^{(n)}=\widetilde{k}_{2}^{(n)}$ at $s_{0}$ for $n \in \mathbb{N} \cup\{0\}$. Since $k_{2}$ and $\widetilde{k}_{2}$ are real analytic, they are identical in an interval containing $s_{0}$. Furthermore, they are the same on $[0, L]$ because the set where two distinct real analytic functions are equal must be discrete.

From now on, we are only interested in the case of nonzero $\kappa$.

\section{The Solutions of Constant Twisting Density}

In this section, $t_{w}$ is a constant. Thus (8) gives $k_{1}=0$. Moreover, (9) and (10) become

$$
\begin{array}{r}
\rho \ddot{k}_{2}+\left((\tilde{\rho}-\rho) k_{2}+\rho \kappa\right) t_{w}^{2}-\tilde{\rho} \tau k_{2} t_{w}-k_{2} \lambda_{3}=0, \\
\left((2 \rho-\tilde{\rho}) t_{w}+\tilde{\rho} \tau\right) \dot{k}_{2}=0,
\end{array}
$$

respectively. The first equation can be further written as

$$
\ddot{k}_{2}=-\frac{1}{2} k_{2}^{3}+\left(\frac{\epsilon}{\rho}+\frac{2 \rho-3 \tilde{\rho}}{2 \rho} t_{w}^{2}+\frac{\tilde{\rho}}{\rho} \tau t_{w}\right) k_{2}-\kappa t_{w}^{2} .
$$

If $k_{2}$ is a constant, then there are at most three choices for that constant since (13) becomes a cubic equation of $k_{2}$. So there are at most three solutions. In fact, each solution gives rise to an elastica whose axis would be a line, a circular arc, or a circular helix; moreover, the geometric torsion of the axis equals $t_{w}$. 
For the rest of this section, $k_{2}$ is assumed nonconstant. Then (12) immediately yields $(2 \rho-\tilde{\rho}) t_{w}+\tilde{\rho} \tau=0$. Multiplying both sides of (13) by $2 \dot{k}_{2}$ and then integrating, one obtains

$$
\left(\dot{k}_{2}\right)^{2}=-\frac{1}{4} k_{2}^{4}+\left(\frac{\epsilon}{\rho}-\left(1+\frac{\tilde{\rho}}{2 \rho}\right) t_{w}^{2}\right) k_{2}^{2}-2 \kappa t_{w}^{2} k_{2}+c_{1}
$$

where $c_{1}$ is a constant of integration.

Let $Q(x)$ be the following quartic polynomial:

$$
Q(x)=x^{4}-4\left(\frac{\epsilon}{\rho}-\left(1+\frac{\tilde{\rho}}{2 \rho}\right) t_{w}^{2}\right) x^{2}+8 \kappa t_{w}^{2} x-4 c_{1} .
$$

Then (14) becomes $\left(\dot{k}_{2}\right)^{2}+Q\left(k_{2}\right) / 4=0$. Consider the discriminant $D$ of $Q(x)$ :

$$
D=D\left(c_{1}\right)=-4096\left(4\left(c_{1}-\frac{1}{3} c_{2}^{2}\right)^{3}+27\left(\frac{2}{3} c_{1} c_{2}-\kappa^{2} t_{w}^{4}+\frac{2}{27} c_{2}^{3}\right)^{2}\right)
$$

where

$$
c_{2}=\frac{\epsilon}{\rho}-\left(1+\frac{\tilde{\rho}}{2 \rho}\right) t_{w}^{2} .
$$

Choosing $c_{1}$ carefully so that it is not a root of $D$, then the roots of $Q(x)$ are mutually distinct. Because $k_{2}$ satisfies (14) and is not constant, $Q(x)$ has to be negative somewhere in $[0, L]$. In addition, $Q(x) \rightarrow+\infty$ as $x \rightarrow \pm \infty$. So $Q(x)$ has real roots. Moreover, the number of real roots is either two when $D<0$, or four when $D>0$. In the former case, we use (259.00) of [9] to solve $k_{2}$ from (14). In the latter case, let $p_{i}$ 's, for $1 \leq i \leq 4$, denote the roots of $Q(x)$ with the ordering $p_{1}>p_{2}>p_{3}>p_{4}$. Then $k_{2}$ can be solved by means of (252.00) or (253.00) of [9] if $p_{4} \leq k_{2} \leq p_{3}$ is assumed, and (256.00) or (257.00) of [9] if $p_{2} \leq k_{2} \leq p_{1}$ is assumed.

At each root $r_{i}$ of $D$, where $1 \leq i \leq m$ and $m$ is either 1 or 3 , there exists a level set $\mathcal{L}_{i} \subset \mathbb{R}^{2}$ of the following function:

$$
\Psi(x, y)=y^{2}+\frac{1}{4} x^{4}-\left(\frac{\epsilon}{\rho}-\left(1+\frac{\tilde{\rho}}{2 \rho}\right) t_{w}^{2}\right) x^{2}+2 \kappa t_{w}^{2} x .
$$

Each $\mathcal{L}_{i}$, if not degenerate, is a real algebraic curve which is possibly singular and consists of at most two components. Let $\left(x_{0}, y_{0}\right)$ be a point of $\mathbb{R}^{2} \backslash\left(\mathcal{L}_{1} \cup \cdots \cup \mathcal{L}_{m}\right)$ so that $(D \circ \Psi)\left(x_{0}, y_{0}\right)<0$, for example, it is very distant from the origin. Since $D \circ \Psi$ is continuous on $\mathbb{R}^{2}$, there is a connected open neighborhood of $\left(x_{0}, y_{0}\right)$, call it $N$, so that $D \circ \Psi<0$ in $N$. Since $\Psi$ is never constant and is continuous in $N, \Psi(N)$ is an infinite subset of $\mathbb{R}$. Because each number of $\Psi(N)$ determines a unique solution through (259.00) of [9], there are infinitely many solutions with the same total energy $\epsilon$ and twisting density $t_{w}$. So, Theorem 1 is proved.

\section{Proof of Theorem 2}

Because of (8), we consider (9) and (10) differential equations of $k_{2}$ with variable coefficients. More precisely, (9) and (10) can be written as

$$
\rho \ddot{k}_{2}-\left(\epsilon-\frac{\rho}{2} k_{2}^{2}-\frac{\tilde{\rho}^{2}}{2 \rho \kappa^{2}}\left(\dot{t}_{w}\right)^{2}+\frac{2 \rho-3 \tilde{\rho}}{2} t_{w}^{2}\right) k_{2}=f\left(t_{w}\right)
$$




$$
\left((2 \rho-\tilde{\rho}) t_{w}+\tilde{\rho} \tau\right) \dot{k}_{2}+(\rho-\tilde{\rho}) \dot{t}_{w} k_{2}-\frac{\tilde{\rho}}{2 \kappa} \dot{t}_{w} k_{2}^{2}=g\left(t_{w}\right)
$$

respectively, where

$$
\begin{aligned}
& f\left(t_{w}\right)=-\frac{\tilde{\rho}}{\rho \kappa}\left((2 \rho-\tilde{\rho}) t_{w}+\tilde{\rho} \tau\right) \ddot{t}_{w}+\frac{\tilde{\rho}(\tilde{\rho}-\rho)}{\rho \kappa}\left(\dot{t}_{w}\right)^{2}-\rho \kappa t_{w}^{2}, \\
& g\left(t_{w}\right)=\frac{\tilde{\rho}}{\kappa} t_{w}^{(3)}-\frac{\tilde{\rho}}{\rho \kappa} \dot{t}_{w}\left(\epsilon-\frac{\tilde{\rho}^{2}}{2 \rho \kappa^{2}}\left(\dot{t}_{w}\right)^{2}+\frac{2 \rho-3 \tilde{\rho}}{2} t_{w}^{2}+\tilde{\rho} \tau t_{w}\right)+\rho \kappa \dot{t}_{w} .
\end{aligned}
$$

Proposition 3 Given a number $\epsilon$ and a function $t_{w}$, there exists a cubic polynomial $P$ of variable coefficients, depending on $\epsilon, \rho, \tilde{\rho}, \kappa, \tau, t_{w}$ and the derivatives of $t_{w}$, so that $P\left(k_{2}\right)=0$ on $[0, L]$, if $\left(\epsilon, k_{2}, t_{w}\right)$ represents a solution to $(8)-(10)$.

Proof For the solution $\left(\epsilon, k_{2}, t_{w}\right)$, using (16) to eliminate the $\dot{k}_{2}$ term occurring in the result of $\rho \times(16)^{\prime}-\left((2 \rho-\tilde{\rho}) t_{w}+\tilde{\rho} \tau\right) \times(15)$, where (16)' denotes the first derivative of (16), one obtains

$$
\xi_{0} k_{2}^{3}+\xi_{1} k_{2}^{2}+\xi_{2} k_{2}+\xi_{3}=0
$$

with

$$
\begin{aligned}
\xi_{0}= & \frac{\tilde{\rho}^{2}}{\kappa^{2}}\left(\dot{t}_{w}\right)^{2}+\left((2 \rho-\tilde{\rho}) t_{w}+\tilde{\rho} \tau\right)^{2}, \\
\xi_{1}= & \frac{\tilde{\rho}}{\kappa}\left(\left((2 \rho-\tilde{\rho}) t_{w}+\tilde{\rho} \tau\right) \ddot{t_{w}}+(4 \tilde{\rho}-5 \rho)\left(\dot{t}_{w}\right)^{2}\right), \\
\xi_{2}= & \frac{2 \tilde{\rho}}{\kappa} g\left(t_{w}\right) \dot{t}_{w}+\frac{\tilde{\rho}^{2}}{\rho^{2} \kappa^{2}}\left((2 \rho-\tilde{\rho}) t_{w}+\tilde{\rho} \tau\right)^{2}\left(\dot{t}_{w}\right)^{2}+2(3 \rho-2 \tilde{\rho})(\rho-\tilde{\rho})\left(\dot{t}_{w}\right)^{2} \\
& -2(\rho-\tilde{\rho})\left((2 \rho-\tilde{\rho}) t_{w}+\tilde{\rho} \tau\right) \dot{t}_{w}-\left((2 \rho-\tilde{\rho}) t_{w}+\tilde{\rho} \tau\right)^{2}\left(\frac{2 \epsilon}{\rho}+\frac{2 \rho-3 \tilde{\rho}}{\rho} t_{w}^{2}\right), \\
\xi_{3}= & 2\left((2 \rho-\tilde{\rho}) t_{w}+\tilde{\rho} \tau\right) \dot{g}\left(t_{w}\right)-\frac{2}{\rho}\left((2 \rho-\tilde{\rho}) t_{w}+\tilde{\rho} \tau\right)^{2} f\left(t_{w}\right)-2(3 \rho-2 \tilde{\rho}) g\left(t_{w}\right) \dot{t}_{w} .
\end{aligned}
$$

That is, if $P(x)$ denotes the cubic polynomial $\xi_{0} x^{3}+\xi_{1} x^{2}+\xi_{2} x+\xi_{3}$, then $P\left(k_{2}\right)=0$ for the solution $\left(\epsilon, k_{2}, t_{w}\right)$.

We now prove Theorem 2. Choose a point of $[0, L]$ arbitrarily, call it $s_{0}$. If there exists a solution $\left(\epsilon, k_{2}, t_{w}\right)$, then, as a root of the cubic polynomial $P$ with the coefficients evaluated at $s_{0}$, there are at most three numbers that $k_{2}\left(s_{0}\right)$ possibly assumes. Except for the case of $2 \rho=\tilde{\rho}$ and $\tau=0$, we may suppose $(2 \rho-\tilde{\rho}) t_{w}+\tilde{\rho} \tau \neq 0$ at $s_{0}$. Then each $k_{2}\left(s_{0}\right)$ determines a unique $\dot{k}_{2}\left(s_{0}\right)$ by means of (16). Therefore, through Proposition 2 we conclude that there are at most three solutions with the total energy $\epsilon$ and twisting density $t_{w}$.

The proof when $2 \rho=\tilde{\rho}$ and $\tau=0$ proceeds as follows. Suppose $\left(\epsilon, k_{2}, t_{w}\right)$ and $\left(\epsilon, k_{2}+u, t_{w}\right)$ are two distinct solutions. Let $J=\left\{s \in[0, L]: \dot{t}_{w}(s)=0\right\}$. Because $k_{2}+u$ satisfies (16), one obtains $u=0$ or $-2 k_{2}-\kappa$ at any point of $[0, L]$ but outside $J$. We may further assume $k_{2} \neq-\kappa / 2$ at some such point, call it $s_{0}$, since otherwise $u$ has already been zero over $[0, L]$ (note, $J$ is finite since $t_{w}$ is not constant by virtue of Proposition 1 ). Next, consider the first derivative of (16):

$$
\rho\left(k_{2}^{2}+\kappa k_{2}\right) \ddot{t}_{w}+\rho\left(2 k_{2}+\kappa\right) \dot{k}_{2} \dot{t}_{w}=-\kappa \dot{g}\left(t_{w}\right) .
$$


By substituting $k_{2}+u$ for $k_{2}$ in the last equation, one obtains

$$
\rho u\left(u+2 k_{2}+\kappa\right) \ddot{t}_{w}+2 \rho u \dot{k}_{2} \dot{t}_{w}+\rho\left(2 u+2 k_{2}+\kappa\right) \dot{t}_{w} \dot{u}=0 .
$$

So $\dot{u}=-2 \dot{k}_{2}$ at $s_{0}$. Therefore, through Proposition 2 we conclude that there are at most two solutions with the total energy density $\epsilon$ and twisting density $t_{w}$.

\section{Discussions}

In the proof of Theorem 2, we also described the difference between two distinct solutions with the same total energy and twisting density, if they exist, when $2 \rho=\tilde{\rho}$ and $\tau=0$. As $s_{0}=0$ is assumed for sake of convenience, it suggests that the solution with the initial data $\left(k_{1}(0), k_{2}(0), t_{w}(0), \dot{k}_{1}(0), \dot{k}_{2}(0)\right)$ and the solution with the initial data $\left(k_{1}(0),-k_{2}(0)-\kappa, t_{w}(0), \dot{k}_{1}(0),-\dot{k}_{2}(0)\right)$ have the same twisting density, provided they have the same total energy. Figure 1 demonstrates the plots of the twisting density and its first and second derivatives of two such solutions. They were produced by a computer program incorporating an ODE solver of MATLAB, called ode45, which implements the Runge-Kutta method.
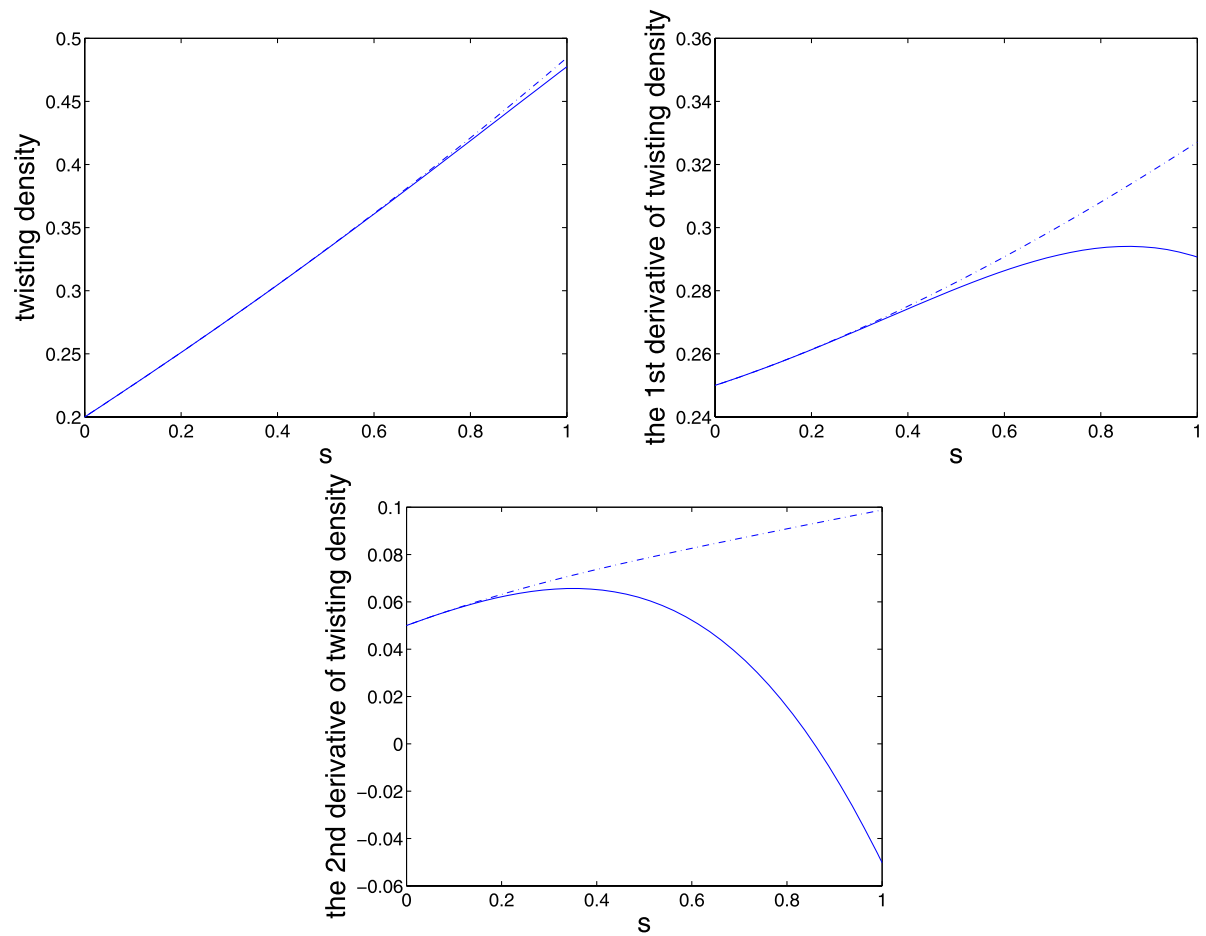

Fig. 1 The initial data for the solution whose twisting density and its first and second derivatives are plotted by solid lines are $k_{1}(0)=0.5, k_{2}(0)=1, t_{w}(0)=0.2, \dot{k}_{1}(0)=0.1, \dot{k}_{2}(0)=0.1$. The initial data for the solution whose twisting density and its first and second derivatives are plotted by dashdot lines are the same as the ones for the solid lines, except $k_{2}(0)=-2, \dot{k}_{2}(0)=-0.1$. Here, we set $\rho=0.5, \tilde{\rho}=1, \epsilon=1, \kappa=1$ and $L=1$, the stepsize is default 
Fig. 2 The top and bottom rods are constructed by using the numerical solutions whose twisting densities were plotted by the solid line and the dashdot line in Fig. 1, respectively. For both rods, $\mathbf{d}_{1}$ is in the lighter gray color and $\mathbf{d}_{2}$ is in the darker gray color
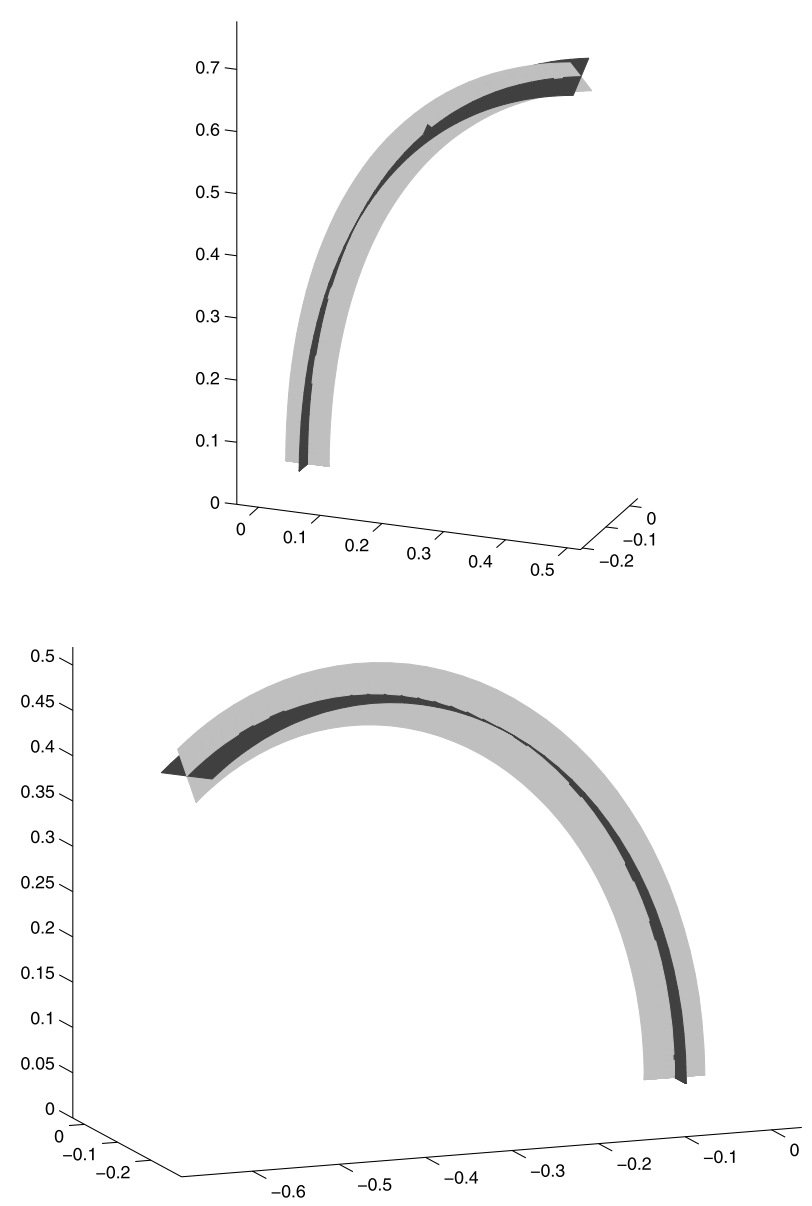

Using the numerical solutions, we can construct the configurations of the two elasticas, see Fig. 2. For simplicity, we set $\sigma_{3}=0$ so that the undeformed state of the rods has zero twisting density. (In Appendix, we shall briefly explain how to construct the configuration of a rod from a solution.)

Clearly, the twisting densities plotted in Fig. 1 are different. To illustrate that the truncation error of Runge-Kutta method is not responsible for the difference, in Fig. 3 we show four plots of $\dot{t}_{w}$ with smaller and smaller stepsizes $h=10^{-2}, 10^{-3}, 10^{-4}$ and $10^{-5}$. We also compare these with the plot of $\dot{t}_{w}$ from Fig. 1 . The readers can easily see that these plots are almost identical one another.

Our numerical computation suggests that there are no two distinct solutions with the same total energy and twisting density when $2 \rho=\tilde{\rho}$ and $\tau=0$. We also acknowledge that the argument used to prove Theorem 2 is not thorough enough to completely confirm the multiplicity of the solutions. So it is reasonable to ask the following question: Is it possible to improve Theorem 2 so that there is exactly one solution for the prescribed total energy and twisting density which is not constant? The next example, however, shows that the desired improvement is impossible in general. 

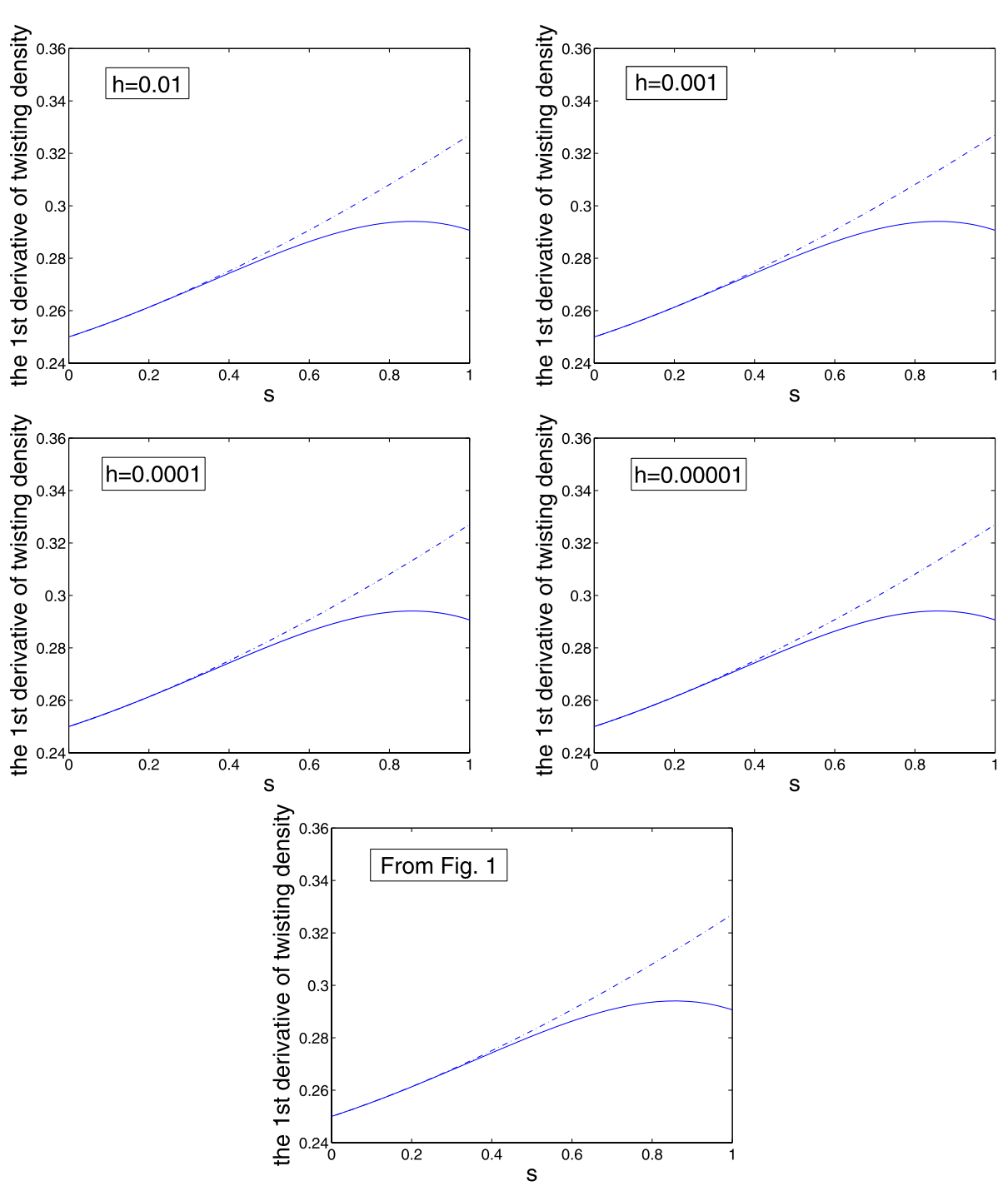

Fig. 3 Plots with different step sizes

Example Let $\left(\epsilon, k_{2}, t_{w}\right)$ denote a force-free solution, namely $\boldsymbol{l}_{\mathcal{R}}=0$, where $t_{w}$ is nonconstant. Using (7) and (8) one gets

$$
k_{2}=\frac{\tilde{\rho}(\tilde{\rho}-\rho)}{2 \rho^{2} \kappa} t_{w}^{2}-\frac{\tilde{\rho}^{2} \tau}{\rho^{2} \kappa} t_{w}+y,
$$

where $y$ is a constant of integration. Seemingly, the above equation gives infinitely many $k_{2}$ 's because of the presence of $y$. But if there are indeed two such $k_{2}$ 's, then (6) yields $(\tilde{\rho}-\rho) t_{w}-\tilde{\rho} \tau=0$ on $[0, L]$. Because $t_{w}$ is not constant, the last equation holds only when $\rho=\tilde{\rho}$ and $\tau=0$. So there is at most one force-free solution with total energy $\epsilon$ and twisting density $t_{w}$, unless $\rho=\tilde{\rho}$ and $\tau=0$. From now on, let us focus on this exceptional case 
Fig. 4 These two rods are constructed by setting $\rho=\tilde{\rho}=1, \kappa=1, L=1, \sigma_{3}=0$, $\epsilon=0.645, A=0.2, B=0.5$, and $y=1$ for the top rod and $y=-1$ for the bottom one. For both rods, $\mathbf{d}_{1}$ is in the lighter gray color and $\mathbf{d}_{2}$ is in the darker gray color
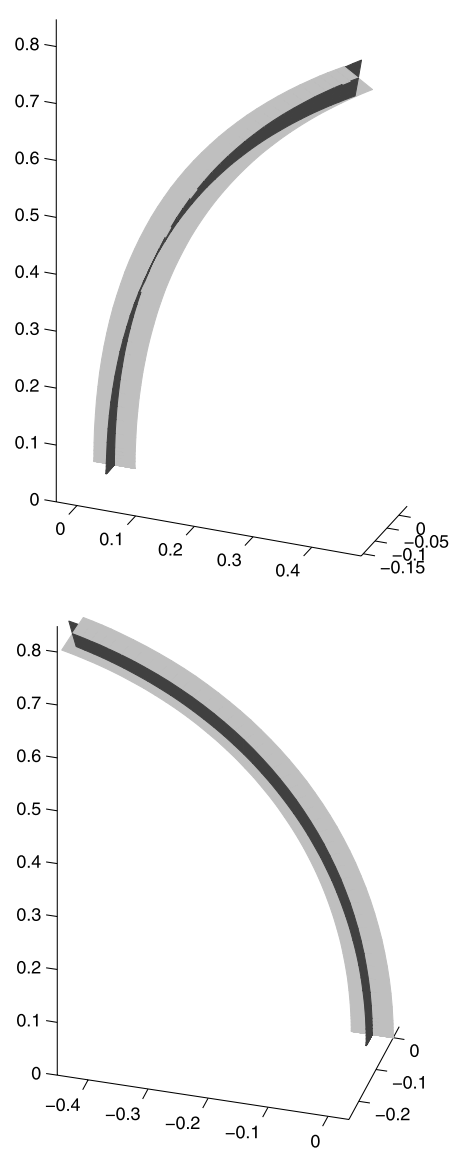

in which $k_{2}$ degenerates into the constant $y$ and (6) becomes $\ddot{t}_{w}+\kappa^{2} t_{w}=0$. It is easy to obtain $t_{w}=A \cos \kappa s+B \sin \kappa s$ where $A$ and $B$ are two constants. Owing to KirchhoffClebsch conservation law [20], the constants $A, B$ satisfy $A^{2}+B^{2}=2 \epsilon / \rho-y^{2}$. As a result, there are two $y$ 's, namely $y= \pm \sqrt{2 \epsilon / \rho-A^{2}-B^{2}}$. Therefore, there are indeed two distinct solutions of the same total energy and nonconstant twisting density. The configurations of such rods are shown in Fig. 4; they are constructed by using $\rho=\tilde{\rho}=1, \kappa=1, L=1, \sigma_{3}=$ $0, \epsilon=0.645, A=0.2$ and $B=0.5$.

Suggested by the preceding example, we therefore restrict ourselves to one of the following cases: (i) $\tau \neq 0$, and (ii) $\tau=0$ and $\rho \neq \tilde{\rho}$, while attempting to answer the question. We are particularly interested in the second case, for the condition $\tau=0$ refers to the naturally circular elastic rods that have been employed to simulate the tertiary structure of DNA with intrinsic curvature; the condition $\rho \neq \tilde{\rho}$ has been applied in many studies employing numerical methods, for example, $\rho=2.70 \times 10^{-19} \mathrm{erg}-\mathrm{cm}$ and $\tilde{\rho}=2.04 \times 10^{-19} \mathrm{erg}$-cm for a DNA molecule in a solution of dilute $\mathrm{NaCl}[34]$.

Acknowledgement This work was partially supported by NSF Grant \#0511766 and by a Sloan Fellowship (L.-T. Cheng), and by NSC (ROC) 89-2119-M-259-002 (K. Hu). The authors would like to thank the referee for the valuable comments. 
Open Access This article is distributed under the terms of the Creative Commons Attribution Noncommercial License which permits any noncommercial use, distribution, and reproduction in any medium, provided the original author(s) and source are credited.

\section{Appendix}

The directors of a rod give rise to a curve in $S O(3)$ starting at the identity matrix $I$, while comparing with $\mathbf{i}, \mathbf{j}, \mathbf{k}$. Through a 2-fold covering map $p: S^{3} \rightarrow S O(3)$, the curve in $S O(3)$ can be lifted to a unique curve in $S^{3}$ starting at $(1,0,0,0)$. Here, the covering map is defined by $p(\mathbf{q})(\mathbf{v})=q^{-1} v q$, where $\mathbf{v}=\left(v_{1}, v_{2}, v_{3}\right) \in \mathbb{R}^{3}$ is any vector that is also identified with a purely imaginary quaternion $v=i v_{1}+j v_{2}+k v_{3}, q=q_{1}+i q_{2}+j q_{3}+k q_{4}$ is a quaternion corresponding to $\mathbf{q}=\left(q_{1}, q_{2}, q_{3}, q_{4}\right) \in S^{3}$, and the expression $q^{-1} v q$ is a product of quaternions $q^{-1}, v$ and $q$ (note, $q^{-1}=\bar{q}=q_{1}-i q_{2}-j q_{3}-k q_{4}$, called the conjugate of $q$, since $\left.\mathbf{q} \in S^{3}\right)$. Because $\mathbf{d}_{1}=p(\mathbf{q})(\mathbf{i}), \mathbf{d}_{2}=p(\mathbf{q})(\mathbf{j})$ and $\mathbf{d}_{3}=p(\mathbf{q})(\mathbf{k})$, we have

$$
\begin{array}{ll}
\mathbf{d}_{1}=\left(\begin{array}{c}
q_{1}^{2}+q_{2}^{2}-q_{3}^{2}-q_{4}^{2} \\
2\left(q_{2} q_{3}-q_{1} q_{4}\right) \\
2\left(q_{1} q_{3}+q_{2} q_{4}\right)
\end{array}\right), \quad \mathbf{d}_{2}=\left(\begin{array}{c}
2\left(q_{1} q_{4}+q_{2} q_{3}\right) \\
q_{1}^{2}-q_{2}^{2}+q_{3}^{2}-q_{4}^{2} \\
2\left(q_{3} q_{4}-q_{1} q_{2}\right)
\end{array}\right), \\
\mathbf{d}_{3}=\left(\begin{array}{c}
2\left(q_{2} q_{4}-q_{1} q_{3}\right) \\
2\left(q_{1} q_{2}+q_{3} q_{4}\right) \\
q_{1}^{2}-q_{2}^{2}-q_{3}^{2}+q_{4}^{2}
\end{array}\right) .
\end{array}
$$

According to the definitions of $u_{i}$ and the fact of $\mathbf{q} \in S^{3}$, we have

$$
\left(\begin{array}{cccc}
q_{2} & -q_{1} & q_{4} & -q_{3} \\
q_{3} & -q_{4} & -q_{1} & q_{2} \\
q_{4} & q_{3} & -q_{2} & -q_{1} \\
q_{1} & q_{2} & q_{3} & q_{4}
\end{array}\right)\left(\begin{array}{c}
\dot{q}_{1} \\
\dot{q}_{2} \\
\dot{q}_{3} \\
\dot{q}_{4}
\end{array}\right)=\left(\begin{array}{c}
\frac{1}{2} u_{1} \\
\frac{1}{2} u_{2} \\
\frac{1}{2} u_{3} \\
0
\end{array}\right),
$$

or equivalently

$$
\left(\begin{array}{c}
\dot{q}_{1} \\
\dot{q}_{2} \\
\dot{q}_{3} \\
\dot{q}_{4}
\end{array}\right)=\left(\begin{array}{cccc}
q_{2} & q_{3} & q_{4} & q_{1} \\
-q_{1} & -q_{4} & q_{3} & q_{2} \\
q_{4} & -q_{1} & -q_{2} & q_{3} \\
-q_{3} & q_{2} & -q_{1} & q_{4}
\end{array}\right)\left(\begin{array}{c}
\frac{1}{2} u_{1} \\
\frac{1}{2} u_{2} \\
\frac{1}{2} u_{3} \\
0
\end{array}\right) .
$$

Our scheme of obtaining the configuration of an equilibrium rod from a solution to the Euler-Lagrange equations is to use (5) to get $u_{i}$ 's from $k_{1}, k_{2}$ and $t_{w}$ (to avoid complexity, our previous demonstrations were done by assuming $\sigma_{3}=0$ which implies $\phi=0$ ), and next apply any numerical method to solve (19) with the initial conditions: $q_{1}(0)=1, q_{2}(0)=$ $q_{3}(0)=q_{4}(0)=0$, and finally integrate $\mathbf{d}_{3}$ to obtain the rod axis.

\section{References}

1. Bates, A.D., Maxwell, A.: DNA Topology. IRL Press, Oxford (1993)

2. Bauer, W.R., Lund, R.A., White, J.H.: Twist and writhe of a DNA loop containing intrinsic bends. Proc. Natl. Acad. Sci. USA 90, 833-837 (1993)

3. Benham, C.J.: Elastic model of supercoiling. Proc. Natl. Acad. Sci. USA 74, 2387-2401 (1977)

4. Benham, C.J.: Geometry and mechanics of DNA superhelicity. Biopolymers 22, 2477-2495 (1983) 
5. Benham, C.J.: Theoretical analysis of conformational equilibria in superhelical DNA. Ann. Rev. Biophys. Biophys. Chem. 14, 23-45 (1985)

6. Benham, C.J.: The role of the stress resultant in determining mechanical equilibria of superhelical DNA. Biopolymers 26, 9-15 (1987)

7. Benham, C.J.: Onset of writhing in circular elastic polymers. Phys. Rev. A 39, 2582-2586 (1989)

8. Birkhoff, G., Rota, G.-C.: Ordinary Differential Equations, 2nd edn. Wiley, New York (1969)

9. Byrd, R.F., Friedman, M.D.: Handbook of Elliptic Integrals for Engineers and Physicists. Springer, Berlin (1954)

10. Coleman, B.D., Olson, W.K., Swigon, D.: Theory of sequence-dependent DNA elasticity. J. Chem. Phys. 118, 7127-7140 (2003)

11. Coleman, B.D., Swigon, D.: Theory of supercoiled elastic rings with self-contact and its application to DNA plasmids. J. Elast. 60, 171-221 (2000)

12. Coleman, B.D., Tobias, I., Swigon, D.: Theory of the influence of end conditions on self-contact in DNA loops. J. Chem. Phys. 103, 9101-9109 (1995)

13. Fain, B., Rudnick, J., Östlund, S.: Conformations of linear DNA. Phys. Rev. E 55, 7364-7368 (1997)

14. Fain, B., Rudnick, J.: Conformations of closed DNA. Phys. Rev. E 60, 7239-7252 (1999)

15. Hagerman, P.J.: Flexibility of DNA. Ann. Rev. Biophys. Biophys. Chem. 17, 265-286 (1988)

16. Hao, M.-H., Olson, W.K.: Global equilibrium configurations of supercoiled DNA. Macromolecules 22, 3292-3303 (1989)

17. Hu, K.: A differential-geometric interpretation of Kirchhoff's elastic rods. J. Math. Phys. 40, 3341-3352 (1999)

18. Hu, K.: Writhe of DNA induced by a terminal twist. Bull. Math. Biol. 67, 197-209 (2005) (with Erratum ibid. 67, 1155 (2005))

19. Le Bret, M.: Catastrophic variation of twist and writhing of circular DNAs with constraint. Biopolymers 18, 1709-1725 (1979)

20. Love, A.E.H.: A Treatise on the Mathematical Theory of Elasticity, 4th edn. Dover, New York (1944)

21. Manning, R.S., Maddocks, J.H., Kahn, J.D.: A continuum rod model of sequence-dependent DNA structure. J. Chem. Phys. 105, 5626-5646 (1996)

22. Marini, J.C., Levene, S.D., Crothers, D.M., Englund, P.T.: A bent helix in kinetoplast DNA. Cold Spring Harbor Symp. Quant. Biol. 47, 279-283 (1982)

23. Olson, W.K.: Simulating DNA at low resolution. Curr. Opin. Struck. Biol. 6, 242-256 (1996)

24. Qian, H., White, J.H.: Terminal twist induced continuous writhe of a circular rod with intrinsic curvature. J. Biomol. Struct. Dyn. 16, 663-669 (1998)

25. Qian, H., White, J.H.: Twist induced abrupt writhe of naturally straight arch with induced curvature (unpublished)

26. Shi, Y., Hearst, J.E.: The Kirchhoff elastic rod, the nonlinear Schrödinger equation, and DNA supercoiling. J. Chem. Phys. 101, 5184-5200 (1994)

27. Swigon, D., Coleman, B.D., Tobias, I.: The elastic rod model for DNA and its application to the tertiary structure of DNA minicircles in mononucleosomes. Biophys. J. 74, 2515-2530 (1998)

28. Tobias, I., Coleman, B.D., Lembo, M.: A class of exact dynamic solutions in the elastic rod model of DNA with implications for the theory of fluctuations in the torsional motion of plasmids. J. Chem. Phys. 105, 2517-2526 (1996)

29. Tobias, I., Coleman, B.D., Olson, W.K.: The dependence of DNA tertiary structure on end conditions: Theory and implications for topological transitions. J. Chem. Phys. 101, 10990-10996 (1994)

30. Tobias, I., Olson, W.K.: The effect of intrinsic curvature on supercoiling: predictions of elasticity theory. Biopolymers 33, 639-646 (1993)

31. Ulanovsky, L., Bodner, M., Trifonov, E.N., Choder, M.: Curved DNA: Design, synthesis, and circularization. Proc. Natl. Acad. Sci. USA 83, 862-866 (1986)

32. Wadati, M., Tsuru, H.: Elastic model of looped DNA. Physica D 21, 213-226 (1986)

33. Watson, J.D., Crick, F.H.C.: Molecular structure of nucleic acids. A structure for deoxyribose nucleic acid. Nature 171, 737-738 (1953)

34. Westcott, T.P., Tobias, I., Olson, W.K.: Elasticity theory and numerical analysis of DNA supercoiling: An application to DNA looping. J. Phys. Chem. 99, 17926-17935 (1995)

35. White, J.H., Bauer, W.R.: Finite-element analysis of the displacement of closed DNA loops under torsional stress. Philos. Trans. R. Soc. A 362, 1335-1353 (2004)

36. White, J.H., Lund, R.A., Bauer, W.R.: Twist, writhe, and geometry of a DNA loop containing equally spaced coplanar bends. Biopolymers 38, 235-250 (1996)

37. White, J.H., Lund, R.A., Bauer, W.R.: Effect of salt-dependent stiffness on the conformation of a stressed DNA loop containing initially coplanar bends. Biopolymers 49, 605-619 (1999)

38. Wu, H.-M., Crothers, D.M.: The locus of sequence-directed and protein-induced DNA bending. Nature 83, 509-513 (1984) 